\title{
Single-source chest-abdomen-pelvis cancer staging on a third generation dual-source CT system: comparison of automated tube potential selection to second generation dual-source CT
}

Clara Park, Tatjana Gruber-Rouh, Doris Leithner, Amelie Zierden, Mortiz H. Albrecht, Julian L. Wichmann, Boris Bodelle, Mohamed Elsabaie, Jan-Erik Scholtz, Moritz Kaup, Thomas J. Vogl and Martin Beeres* (D)

\begin{abstract}
Background: Evaluation of latest generation automated attenuation-based tube potential selection (ATPS) impact on image quality and radiation dose in contrast-enhanced chest-abdomen-pelvis computed tomography examinations for gynaecologic cancer staging.

Methods: This IRB approved single-centre, observer-blinded retrospective study with a waiver for informed consent included a total of 100 patients with contrast-enhanced chest-abdomen-pelvis CT for gynaecologic cancer staging. All patients were examined with activated ATPS for adaption of tube voltage to body habitus. 50 patients were scanned on a third-generation dual-source CT (DSCT), and another 50 patients on a second-generation DSCT. Predefined image quality setting remained stable between both groups at $120 \mathrm{kV}$ and a current of 210 Reference mAs.

Subjective image quality assessment was performed by two blinded readers independently. Attenuation and image noise were measured in several anatomic structures. Signal-to-noise ratio (SNR) was calculated. For the evaluation of radiation exposure, $\mathrm{CT}$ dose index ( $\mathrm{CTDI_{ \text {vol } }}$ ) values were compared.

Results: Diagnostic image quality was obtained in all patients. The median CTDI $\mathrm{vol}_{\text {( }}(6.1 \mathrm{mGy}$, range 3.9-22 mGy) was $40 \%$ lower when using the algorithm compared with the previous ATCM protocol (median $10.2 \mathrm{mGy} \cdot \mathrm{cm}$, range 5.8-22.8 $\mathrm{mGy}$ ). A reduction in potential to $90 \mathrm{kV}$ occurred in 19 cases, a reduction to $100 \mathrm{kV}$ in 23 patients and a reduction to $110 \mathrm{kV}$ in 3 patients of our experimental cohort. These patients received significantly lower radiation exposure compared to the former used protocol.
\end{abstract}

Conclusion: Latest generation automated ATPS on third-generation DSCT provides good diagnostic image quality in chest-abdomen-pelvis CT while average radiation dose is reduced by $40 \%$ compared to former ATPS protocol on second-generation DSCT.

Keywords: Multidetector Computed Tomography, Cancer, Cancer Staging, Neoplasms, Automated Tube Potential Selection, Dual-Source CT

\footnotetext{
*Correspondence: beeres@gmx.net

Department of Diagnostic and Interventional Radiology, Clinic of the Goethe

University, Haus 23C UG, Theodor-Stern-Kai 7, 60590 Frankfurt, Germany
} 


\section{Background}

Contrast-enhanced chest-abdomen-pelvis computed tomography $(\mathrm{CT})$ is the standard imaging procedure for oncologic staging. It is widely available and allows evaluation of several anatomic structures such as lymph nodes, abdominal organs, lung and bony structures to detect malignancies in one comprehensive examination. Due to the increased amount of overall CT examinations $[1,2]$, radiation exposure should be used with care following the 'as low as reasonably achievable' (ALARA) rule. Nevertheless, cancer might be life-limiting and cancer staging overweight risks of radiation exposure [3-6]. However, patients with a detected malignancy in an early stage might receive multiple follow-up CT scans with an increased cumulative radiation dose and, therefore, an increased risk of radiation dose related disease [4].

Many techniques have been introduced to lower radiation exposure such as automated attenuation-based tube current and voltage modulation, noise reduction filters, and iterative reconstruction algorithms [2, 6-15]. Automated attenuation based tube potential selection (ATPS) is routinely used in our department for oncologic staging and follow-up chest-abdomen-pelvis CT $[16,17]$.

The aim of our study is to evaluate image quality and radiation exposure of latest generation automated tube potential selection on a third-generation dual-source CT (DSCT) in comparison to the second-generation DSCT in gynaecologic oncology staging and follow up.

\section{Methods}

\section{Patients}

This retrospective study was performed as a singlecentre, observer-blinded study. The institutional review board approved this study; written informed consent requirement was waived. A total of 100 patients (100 women, median age 62.5 [range 31-86 years]) underwent contrast-enhanced chest-abdomen-pelvis CT examinations for gynaecologic oncology staging between October 2013 and March 2016. General exclusion criteria for contrast-enhanced CT included impaired renal function (estimated glomerular filtration rate $<60 \mathrm{~mL} /$ min, calculated by creatinine blood level and patient age), hyperthyroidism, as well as hypersensitivity to iodine contrast media. Non-contrast scans were excluded.

In Group 150 patients were examined on a secondgeneration 128-slice DSCT (Somatom Definition Flash, Siemens Healthcare, Forchheim, Germany) using former generation of ATPS (CAREkV, Siemens, Forchheim, Germany) with steps of $20 \mathrm{kV}$ between $80 \mathrm{kV}$ and $140 \mathrm{kV}$. Group 2 included 50 patients who were examined on a third-generation 192-slice DSCT (SOMATOM Force, Siemens Healthcare) with the latest generation of ATPS which allows tube potential selection in steps of $10 \mathrm{kV}$ between $70 \mathrm{kV}$ and $150 \mathrm{kV}$. Both imaging protocols were adjusted to a pre-defined image quality of $120 \mathrm{kV} / 210$ Ref.mAs (Table 1). Patient populations were paired regarding age, body size and habitus.

\section{Automated attenuation-based $\mathrm{kV}$ selection}

ATPS selects the optimal tube potential based on the selected body region, the scout and a 12-point scale which allows manual selection of desired examination type to optimize for CT-angiography, parenchymal contrastenhanced CT or visualization of bony structures. [17]. This is useful, because for a high-contrast situation used in CT-angiography, lowering of the $\mathrm{kV}$ leads to higher absorption and higher attenuation values of the vascular structures with additional possibility to safe radiation dose in low-kV CT scans. In this study we set focus to an optimal contrast-enhanced parenchymal organ scan for cancer staging.

The automatically selected $\mathrm{kV}$ remained stable throughout the CT scan. In addition, a real-time automatic mAsmodulation software (CARE Dose 4D, Siemens Healthcare) was used to further reduce radiation exposure.

\section{CT examination}

Two hours before the actual CT examination patients were told to start drinking oral contrast media (Micropaque, Guerbet, Villepinte, France) for improved gastrointestinal delineation. Before the start of the CT scan, an intravenous contrast material bolus, containing $1 \mathrm{ml} / \mathrm{kg}$ of iodinated contrast material (Ultravist 370, Bayer-Schering, Germany), followed by a saline chaser of $40 \mathrm{~mL}$ was injected at $2 \mathrm{~mL} / \mathrm{s}$ flow rate via an antecubital vein using a double-syringe power injector (CT2, Medtron, Saarbruecken, Germany).

A fixed delay of $70 \mathrm{~s}$ post injection was used in order to obtain venous contrast. All CT examinations were performed in a cranio-caudal direction starting from the upper thorax aperture down to the femoral ligaments. In

Table 1 Study population and evaluation of examination parameters

\begin{tabular}{lll}
\hline & Group 1 & Group 2 \\
\hline Imaging mode & Single-source & Single-source \\
Machine & Definition Flash & Definition Force \\
Slice $\times$ collimation & $128 \times 0.6$ & $192 \times 0.6$ \\
Pitch & 1.2 & 1.2 \\
kV/ref. mAs & $120 / 210$ (CarekV) & $120 / 210$ (CareDose4D) \\
Patients $90 \mathrm{kV}$ & & 19 \\
Patients $100 \mathrm{kV}$ & 30 & 23 \\
Patients $110 \mathrm{kV}$ & & 3 \\
Patients $120 \mathrm{kV}$ & 16 & 4 \\
Patients $130 \mathrm{kV}$ & & 1 \\
Patients $140 \mathrm{kV}$ & 4 & \\
Patients $150 \mathrm{kV}$ & &
\end{tabular}


group 1 at a collimation of $128 \cdot 0.6 \mathrm{~mm}$, pitch 1.2 , and rotation time of $0.5 \mathrm{~s}$, in group 2 at a collimation of $192 \cdot 0.6 \mathrm{~mm}$, pitch 1.2 , and rotation time of $0.5 \mathrm{~s}$.

\section{CT data reconstruction}

For fast overviewing, images were reconstructed in 5-mm slice thickness with a 5-mm increment. For detailed evaluation, data were additionally reconstructed with a slice thickness of $3 \mathrm{~mm}$ and increment of $1.5 \mathrm{~mm}$ using a medium-smooth soft-tissue convolution kernel (B30f) for parenchymal analysis and a hard convolution kernel for the analysis of bony structures and the lungs (B70f). To allow an image comparison independently of different available iterative reconstruction algorithms (Safire on $2^{\text {nd }}$ generation DSCT, ADMIRE on $3^{\text {rd }}$ generation DSCT, Siemens Healthcare), we selected filtered-back projection (FBP) reconstruction algorithms by purpose for both groups.

\section{Radiation dose estimations}

$\mathrm{CTDI}_{\mathrm{vol}}$ and DLP values displayed in the patient's protocol were recorded.

\section{Image quality}

Subjective image quality was assessed by two radiologists individually with 4 and 7 years of experience in whole body imaging on a five-point rating scale : 1 = excellent: excellent definition of tumour and/or metastases, excellent delineation of the structures; 2 = good: good definition of tumour and/or metastases, minimal image noise; $3=$ adequate: adequate definition of tumour and/or metastases, slight impact of image noise, sufficient for diagnosis; $4=$ poor: poor definition of tumour and/or metastases, low attenuation and difficult delineation of the structures, increased image noise, diagnostic confidence reduced; $5=$ unacceptable/nondiagnostic. The most probable reasons for reduced image quality such as obesity, motion, metallic artefacts, contrast medium flow-related, and contrast timing were noted.

Objective image quality analysis was performed by one radiologist with 7 years of experience in general radiology on a regular PACS workstation (Centricity 4.2, General Electric Healthcare, Munich, Germany). Regions of interest (ROI) were drawn in several anatomic regions such as aorta at the level of the pulmonary trunk, both lobes of the lung, left lobe of the liver, right lobe of the liver, pancreas, spleen, kidneys, gluteus maximus muscle, pelvic bone and pre-sternal in the air at the level of the pulmonary trunk. Attenuation and standard deviation of each ROI were noted. Each measurement was performed four times to minimize bias. Average values for attenuation and standard deviation (SD) of each region were calculated. Background noise was determined as the standard deviation (SD) of the ROI in the air. Signal-to-noise ratio (SNR) was determined according to the following equation: $\mathrm{SNR}=$ attenuation/background noise (Table 3).

Tumour and/or metastases were evaluated and measured using tumour diameters for objective evaluation and, for subjective scoring, we evaluated whether the imaging modality might have affected the tumour staging.

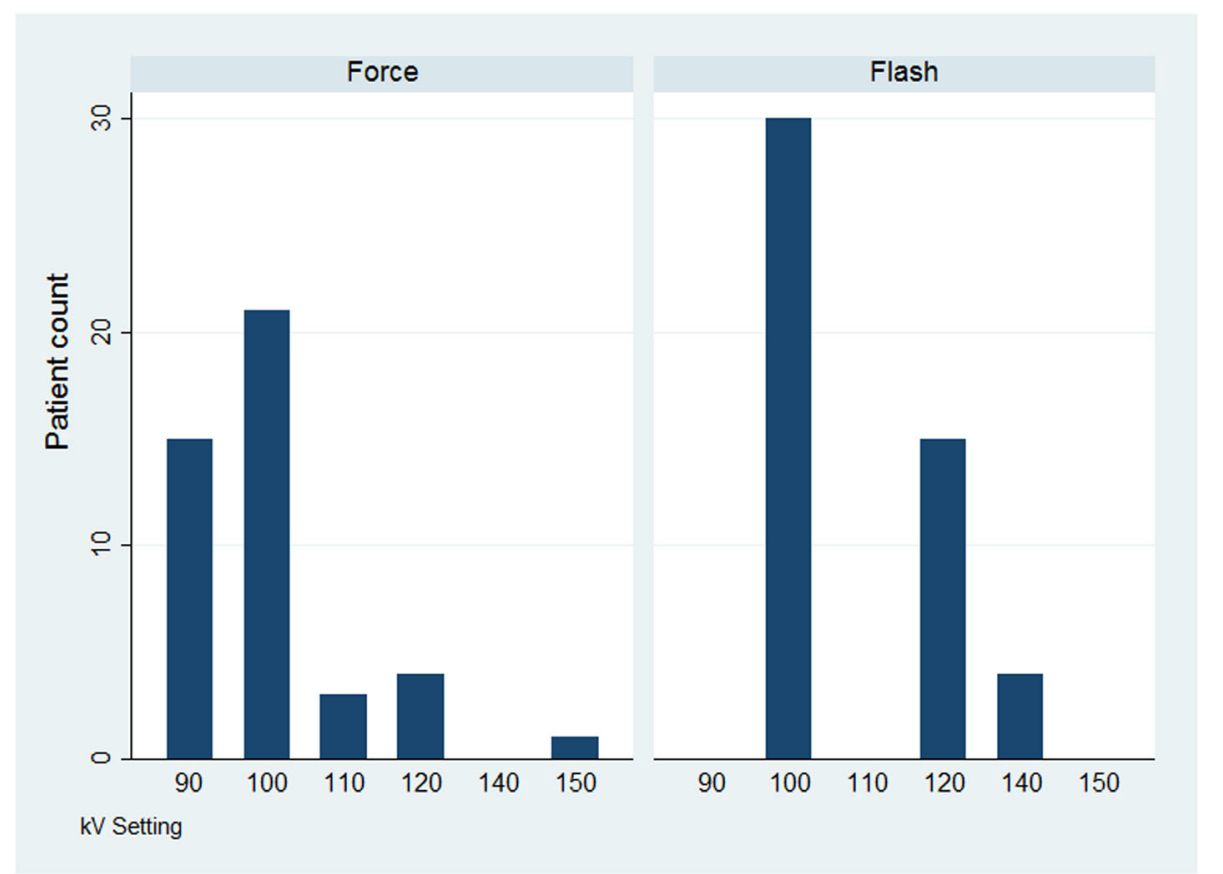

Fig. 1 Choosen kV between both groups 


\section{Statistical analysis}

Statistical analysis was performed using dedicated software (Stata/IC 13, Stata Corp, College Station, Texas, USA). Continuous variables were reported as median and range, categorical variables as frequencies or percentages.

Radiation parameters and quantitative image parameters (e.g. noise, attenuation) were tested using the Wilcoxon Mann-Whitney $U$ test as the data were nonparametric. The relationship between patient diameter and automated $\mathrm{kV}$ selection was analysed using the Spearman rank order correlation test. The Chi-square $(X 2)$ test was used for categorical variables (demographic patient data). Statistical significance was defined as a p-value above 0.05. Differences between the both readers were assessed using Cohen's kappa (j) interpreted in the following way: $j \backslash 0.20$, slight agreement; $j=0.21-0.40$, fair agreement; $j=0.41-$ 0.60 , moderate agreement; $j=0.61-0.80$, substantial agreement; $\mathrm{j}=0.81-1.0$, almost perfect agreement.

\section{Results}

\section{Radiation dose estimation}

In group 1 ATPS resulted in a tube voltage selection of $100 \mathrm{kV}$ in 30 patients, $120 \mathrm{kV}$ in 16 patients and $140 \mathrm{kV}$ in 4 patients. ATPS resulted in a tube voltage reduction to $90 \mathrm{kV}$ in 19 cases, $100 \mathrm{kV}$ in 23 patients, and $110 \mathrm{kV}$ in 3 patients in group 2. 4 Patients were examined at $120 \mathrm{kV}$ and 1 patient at $150 \mathrm{kV}$ (Fig. 1, Table 1).

The median CTDIvol in group 2 was 4.8 mGy (3.9$9.8 \mathrm{mGy})$ at $90 \mathrm{kV}, 6.3 \mathrm{mGy}(5.3-9.0 \mathrm{mGy})$ at $100 \mathrm{kV}$, $9.7 \mathrm{mGy}(6.6-14.1 \mathrm{mGy})$ at $110 \mathrm{kV}, 11.5$ (7.8-14.4 mGy) at $120 \mathrm{kV}$ and $22.0 \mathrm{mGy}$ (only one patient) at $150 \mathrm{kV}$. In the baseline group the median CTDIvol was $8.9 \mathrm{mGy}$ (5.8-14.6 mGy) at $100 \mathrm{kV}, 13.2 \mathrm{mGy}(7.1-17.4 \mathrm{mGy})$ at $120 \mathrm{kV}$ and $20.9 \mathrm{mGy}(20.1-22.8 \mathrm{mGy})$ at $140 \mathrm{kV}$.

The overall comparison between both groups revealed a CTDIvol of $6.1 \mathrm{mGy}(3.9-22.0 \mathrm{mGy})$ in the group 2 and a CTDIvol of $10.2 \mathrm{mGy}(5.9-22.8 \mathrm{mGy})$ in group 1 . Comparing both groups revealed a statistical significant difference with a p-value $<0.01$ (Table 2).

Median lateral patient diameter was $36.8 \mathrm{~cm}$ (30.7$47.4 \mathrm{~cm})$ in group 1 in comparison to $37.2 \mathrm{~cm}(32.2-$ $47.5 \mathrm{~cm})$ in group $2(p=0.2$, Tables 3 and 4$)$. Transversal patient diameter was similar in group 1 (mean, $21.3 \mathrm{~cm}$; range, $12.2-33.0 \mathrm{~cm}$ ) compared to group 2 (mean, $21.8 \mathrm{~cm}$; range $17.3-33.1 \mathrm{~cm} ; p=0.4$ ) (Table 3 ).

\section{Image quality}

Diagnostic image quality was obtained from all patients in group 2 (excellent: $n=47$; good: $n=2$; moderate: $n=$ $1)$. The reasons for moderate image quality were discussed between both readers and, in all cases, due to difficulties in ruling out parenchymal lesions, some because of image noise, and some because of insufficiencies concerning the venous contrast enhancement.
Table 2 Examination parameters

\begin{tabular}{llll}
\hline & Group 1 & Group 2 & $\begin{array}{l}\text { p-Value: } \\
\text { Group 1 } \\
\text { vs. Group 2 }\end{array}$ \\
\hline Patients (all female) & 50 & 50 & \\
Age (years) & & & \\
Scanning range (cm) & $63.9(42.4-75.3)$ & $66.1(41.3-78.1)$ & 0.2 \\
CTDI & $10.2(5.8-22.8)$ & $6.1(3.9-22.0)$ & 0.01 \\
$\begin{array}{l}\text { Dose-length product } \\
\text { (mGy } \times \text { cm) }\end{array}$ & $684(420-1399)$ & $376.2(209.1-1406.7)$ & 0.01 \\
\hline
\end{tabular}

Concerning image quality in the control group, it was also rated as sufficient in all cases (excellent: $n=44$ good: $n=5$; moderate: $n=1$ ).

Inter-reader agreement on measurements was almost perfect $(j=0.82)$.

In summary, the image quality rating demonstrated, that all malignancies could be ruled out adequately and classified in a correct manner with respect to RECIST (Response Evaluation Criteria In Solid Tumours), without statistically significant differences between the two groups $(p=0.4$, Figs. 2 and 3) [18].

\section{Discussion}

Our results showed a reduction in the median $\mathrm{CTDI}_{\mathrm{vol}}$ of $40 \%$ when using latest generation ATPS compared with the previous ATPS protocol. One of the first studies on ATCM performed by Winklehner et al. [19] evaluated automated attenuation-based $\mathrm{kV}$ selection in 40 patients for CT angiography of the aorta. In this study, an overall radiation dose reduction of $25.1 \%$ was observed, while keeping the image quality stable, when using a $120 \mathrm{kV}$ protocol.

Eller et al. [20] evaluated automated attenuation-based $\mathrm{kV}$ selection in 100 patients. They carried out an abdominal CT examination for 52 of the patients, and a thoraco-abdominal examination for 48 patients. All examinations using automated attenuation-based kV selection resulted in a radiation dose reduction of at least $11.4 \%$; in detail, $13.2 \%$ in the abdominal CT group and $9.5 \%$ in the thoraco-abdominal group.

Table 3 Study population - detailed overview

\begin{tabular}{lllc}
\hline & Group 1 & Group 2 & $\begin{array}{l}\text { p-Value: } \\
\text { Group 1 vs. } \\
\text { Group 2 }\end{array}$ \\
\hline Image noise & $7.4(4.4-40.8)$ & $7.9(3.5-23.1)$ & 0.8 \\
SNR & $25.2(4.6-38.5)$ & $27.1(4.3-49.5)$ & 0.08 \\
$\begin{array}{l}\text { Patient diameter } \\
\text { lateral }\end{array}$ & $36.8(30.7-47.4)$ & $37.2(32.2-47.5)$ & 0.2 \\
Patient diameter & $21.3(12.2-33.0)$ & $21.8(17.3-33.1)$ & 0.4 \\
transversal & & & \\
\hline
\end{tabular}


Table 4 Correlation Analysis - Body Diameter/Radiation Dose (the asterisk* marked part should be evaluated with caution because of low patient count)

\begin{tabular}{|c|c|c|c|c|c|c|c|c|}
\hline & & Patients & CTDlvol (mGy) & Total mAs & $\begin{array}{l}\text { Body Diam. } \\
\text { sag }\end{array}$ & $\begin{array}{l}\text { Body diam. } \\
\text { Transv. }\end{array}$ & $\begin{array}{l}\text { Spearman correlation } \\
\text { coefficient (rho) - } \\
\text { lateral diameter/ } \\
\text { CTDlvol }\end{array}$ & $\begin{array}{l}\text { Spearman correlation } \\
\text { coefficient (rho) - } \\
\text { transverse diameter/ } \\
\text { CTDlvol }\end{array}$ \\
\hline \multirow[t]{3}{*}{ Group 1} & $\begin{array}{l}\text { Patients } \\
100 \mathrm{kV}\end{array}$ & 30 & $8.9(5.8-14.6)$ & 217 (179-355) & $32.5(27.1-41.2)$ & $21.2(14.9-30.4)$ & 0.80 & 0.89 \\
\hline & $\begin{array}{l}\text { Patients } \\
120 \mathrm{kV}\end{array}$ & 16 & $10.8(7.1-17.4)$ & $213(152-257)$ & $34.1(27.9-45.8)$ & $24.4(16.7-35.1)$ & 0.81 & 0.83 \\
\hline & $\begin{array}{l}\text { Patients } \\
140 \mathrm{kV}\end{array}$ & 4 & $20.9(20.1-22.8)$ & $218(204-232)$ & $43.8(39.8-48.4)$ & $29.5(24.7-31.5)$ & $0.65^{*}$ & $0.32^{*}$ \\
\hline \multirow[t]{5}{*}{ Group 2} & $\begin{array}{l}\text { Patients } \\
90 \mathrm{kV}\end{array}$ & 19 & $4.8(3.9-9.8)$ & $165.0(134.0-338.0)$ & $34.5(31.9-39.0)$ & $20.4(18.2-24.3)$ & 0.71 & 0.43 \\
\hline & $\begin{array}{l}\text { Patients } \\
100 \mathrm{kV}\end{array}$ & 23 & $6.3(5.3-9.0)$ & $156.0(130.0-224.0)$ & $38.0(32.3-44.1)$ & $21.6(12.2-27.3)$ & 0.68 & 0.71 \\
\hline & $\begin{array}{l}\text { Patients } \\
110 \mathrm{kV}\end{array}$ & 3 & $9.7(6.6-14.1)$ & $185.0(125.0-270.0)$ & $40.4(30.7-47.4)$ & $22.7(17.6-33.0)$ & $1.0 *$ & $1.0 *$ \\
\hline & $\begin{array}{l}\text { Patients } \\
120 \mathrm{kV}\end{array}$ & 4 & $11.5(7.8-14.4)$ & $171.0(106.0-189.0)$ & $37.8(33.4-41.4)$ & $23.1(18.9-28.0)$ & $0.2 *$ & $1.0 *$ \\
\hline & $\begin{array}{l}\text { Patients } \\
150 \mathrm{kV}\end{array}$ & 1 & 22.01 & 192.0 & 42.2 & 28.7 & N/A & N/A \\
\hline
\end{tabular}

Gnannt et al. [21] assessed automatic attenuationbased $\mathrm{kV}$ selection in 40 patients suffering from testicular cancer. In this study, a CT scan of the chest was performed in a mixed arterio-venous phase and the abdominal CT examination was carried out in the portal-venous phase of enhancement. The overall dose reduction was $12 \%$ on average.

With focus on cancer staging, Beeres et al. evaluated automated tube potential selection in 110 patients compared to a $120 \mathrm{kV}$ automated tube potential selection protocol [17]. The overall dose reduction was $7.9 \%$ in a randomly chosen cohort.

In all the studies mentioned above, there was no statistically significant worsening of subjective image quality. In the study by Beeres et al. [17], there was a dose reduction of $7.9 \%$, this is comparable to the study of Eller et al. [20] where there was a dose reduction of $9.5 \%$ in chest-abdomen-pelvis CT examinations reported.
A recently published study of Scholtz et al. compared ATPS on a second-generation DSCT with FBP and third-generation DSCT in combination with a new advanced modelled iterative reconstruction algorithm resulting in an average overall dose reduction of $34.9 \%$ [16]. However, in our study we used FBP in both groups to compare the plain dataset and to prevent from influence of iterative reconstruction algorithm.

Our study showed an overall dose reduction in $40 \%$ of the study population which meets with the study above. The main driver in this dose reduction setting might be the possibility of the tube to select the potential in $10 \mathrm{kV}$ steps compared to the former software. The tube potential in our study switched only once to $150 \mathrm{kV}$ compared with the cited studies, and a tube potential of $80 \mathrm{kV}$ or below wasn't automatically chosen in any case (Table 1 ).

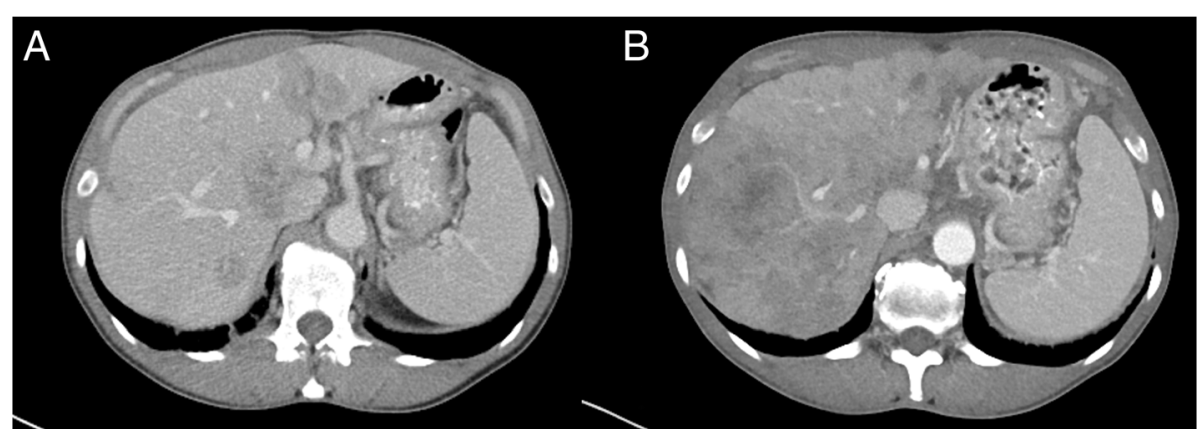

Fig. 2 Image quality comparison in parenchymal liver lesions. Same patient in progressive disease. a Flash-CT: CTDIvol 14.40 mGy, DLP 1035 mGy . cm. b Force-CT: CTDlvol $13.35 \mathrm{mGy}$, DLP $946 \mathrm{mGy} \cdot \mathrm{cm}$ 


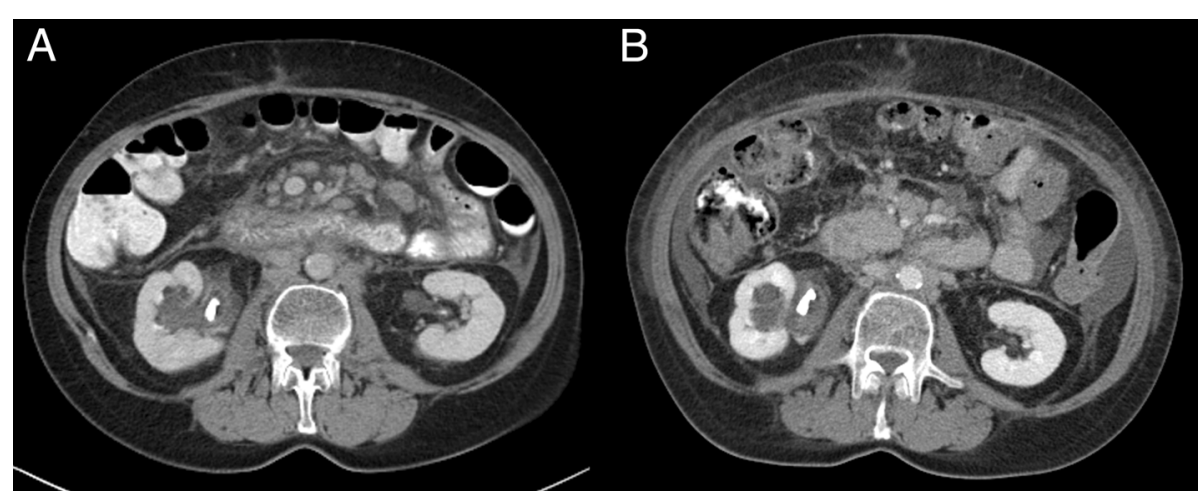

Fig. 3 Image quality comparison in para-aortic lymph-nodes. Same patient in progressive disease. a Flash-CT: CTDlvol 7.72 mGy, DLP 516 mGy. cm. b Force-CT: CTDlvol $3.88 \mathrm{mGy}$, DLP $278.5 \mathrm{mGy} \cdot \mathrm{cm}$

In vascular imaging, it is possible to examine the region of interest using a lower $\mathrm{kV}$ setting because of the high-contrast situation attained by the arterial phase of the contrast material. In contrast, when parenchymal contrast is the clinical setting and the object in question, for example, when liver lesions have to be ruled out, automated attenuation-based tube potential selection might not lower the $\mathrm{kV}$ in the same aggressive manner as in vascular imaging (Fig. 1).

Applying the new algorithm combined with an $\mathrm{x}$-ray tube system that is able to switch between different $\mathrm{kV}$ levels in $10 \mathrm{kV}$ steps, an overall radiation dose reduction of $40 \%$ was possible.

\section{Limitations}

Some limitations of our study need to be addressed. First, the overall number of patients in our study was small; further studies with a larger cohort are required.

Second, we did not record the body mass index, but measured patient diameter as an alternative. Third, while a patient cohort of 100 patients is sufficient for an initial experience, additional larger studies are necessary to assess further potential of this technique.

Fourth, we did not investigate iterative reconstruction algorithms and the effects of ATCM and iterative reconstructions in our study. However, this has been evaluated in prior studies $[16,22]$.

\section{Conclusion}

Our results demonstrate that ATPS in third-generation DSCT allows tube voltage selection in steps of $10 \mathrm{kV}$ resulting in an average dose reduction of $40 \%$ compared to second-generation DSCT and is feasible for oncologic chest-abdomen-pelvis CT examination in clinical routine while overall image quality remains excellent.

\section{Abbreviations}

ALARA: As low as reasonably achievable; ATCM: Automated tube current modulation; ATPS: Automated attenuation-based tube potential selection;
CT: Computed tomography; CTDlvol: CT dose index; DLP: Dose-lengthproduct; kV: Kilovolt; mAs: Miliampere-second; SNR: Signal-to-noise ratio

\section{Acknowledgements}

No Acknowledgements.

Funding

No funding was received for this work.

Availability of data and materials

Not applicable.

\section{Authors' contributions}

Study concepts: MB, CP, TGR, JES. Study design: CP, DL, MA, JLW. Data acquisition: $M B, C P, T J V$. Quality control of data and algorithms: $M B, B B, M K$ Data analysis and interpretation: CP, MB, AZ, JLW. Statistical analysis: MB, JES. Manuscript preparation: MB, CP, AZ, TJV. Manuscript editing: $M B, B B, M E$, MK, AZ. Manuscript review: JES, MB, CP, TGR, DL, MA. All authors read and approved the final manuscript.

Competing interests

The authors declare that they have no competing interests.

\section{Consent for publication}

Each author has participated sufficiently in the submission and takes public responsibility for its content.

\section{Ethics approval and consent to participate}

The institutional review board approved this study (Ethics committee of the Goethe-University); written informed consent requirement was waived since CAREKV is routinely used in all patients undergoing clinically indicated $C T$ in our department

Received: 9 August 2016 Accepted: 3 October 2016 Published online: 10 October 2016

\section{References}

1. Albert JM. Radiation Risk From CT: Implications for Cancer Screening. AJR Am J Roentgenol. 2013;201:W81-87.

2. Shah KH, Slovis BH, Runde D, Godbout B, Newman DH, Lee J. Radiation exposure among patients with the highest $C T$ scan utilization in the emergency department. Emerg Radiol. 2013;20:485-91.

3. Saika K, Machii R. Five-year Relative Survival Rate of Cancer in the USA, Europe and Japan. Jpn J Clin Oncol. 2013;43:1053-4.

4. Cancer statistics. JAMA: the journal of the American Medical Association. 2013;310:982.

5. Brenner DJ, Hall EJ. Computed tomography-an increasing source of radiation exposure. N Engl J Med. 2007;357:2277-84.

6. Bucher AM, Kerl MJ, Albrecht MH, Beeres M, Ackermann H, Wichmann JL, Vogl TJ, Bauer RW, Lehnert T. Systematic Comparison of Reduced Tube Current 
Protocols for High-pitch and Standard-pitch Pulmonary CT Angiography in a Large Single-center Population. Acad Radiol. 2016;23:619-27.

7. Fleischmann D, Boas FE. Computed tomography-old ideas and new technology. Eur Radiol. 2011;21:510-7.

8. Greess H, Nomayr A, Wolf H, Baum U, Lell M, Bowing B, Kalender W, Bautz WA. Dose reduction in $C T$ examination of children by an attenuation-based on-line modulation of tube current (CARE Dose). Eur Radiol. 2002;12:1571-6.

9. Gies M, Kalender WA, Wolf H, Suess C. Dose reduction in $\mathrm{CT}$ by anatomically adapted tube current modulation. I. Simulation studies. Med Phys. 1999;26:2235-47.

10. Kalender WA, Wolf $\mathrm{H}$, Suess C. Dose reduction in CT by anatomically adapted tube current modulation. II. Phantom measurements. Med Phys. 1999;26:2248-53.

11. Kalra MK, Maher MM, Toth TL, Schmidt B, Westerman BL, Morgan HT, Saini S. Techniques and applications of automatic tube current modulation for CT. Radiology. 2004;233:649-57.

12. McCollough CH, Bruesewitz MR, Kofler Jr JM. CT dose reduction and dose management tools: overview of available options. Radiographics. 2006;26:503-12.

13. Scholtz JE, Wichmann JL, Husers K, Albrecht MH, Beeres M, Bauer RW, Vogl $\mathrm{T}$, Bodelle B. Third-generation dual-source $\mathrm{CT}$ of the neck using automated tube voltage adaptation in combination with advanced modeled iterative reconstruction: evaluation of image quality and radiation dose. Eur Radiol. 2016;26:2623-31.

14. Frellesen C, Stock W, Kerl JM, Lehnert T, Wichmann JL, Nau C, Geiger E, Wutzler S, Beeres M, Schulz B, et al. Topogram-based automated selection of the tube potential and current in thoraco-abdominal trauma $\mathrm{CT}$ - a comparison to fixed kV with mAs modulation alone. Eur Radiol. 2014;24:1725-34.

15. Scholtz JE, Husers K, Kaup M, Albrecht MH, Beeres M, Bauer RW, Schulz B, Vogl TJ, Wichmann JL. Evaluation of image quality and dose reduction of 80 $\mathrm{kVp}$ neck computed tomography in patients with suspected peritonsillar abscess. Clin Radiol. 2015;70:e67-73.

16. Scholtz JE, Wichmann JL, Husers K, Beeres M, Nour-Eldin NE, Frellesen C, Vogl TJ, Lehnert T. Automated tube voltage adaptation in combination with advanced modeled iterative reconstruction in thoracoabdominal thirdgeneration 192-slice dual-source computed tomography: effects on image quality and radiation dose. Acad Radiol. 2015;22:1081-7.

17. Beeres M, Romer M, Bodelle B, Lee C, Gruber-Rouh T, Mbalisike E, Kerl JM, Wichmann JL, Schulz B, Vogl TJ, Bauer RW. Chest-abdomen-pelvis $\subset$ for staging in cancer patients: dose effectiveness and image quality using automated attenuation-based tube potential selection. Cancer Imaging. 2014;14:28.

18. Eisenhauer EA, Therasse P, Bogaerts J, Schwartz LH, Sargent D, Ford R, Dancey J, Arbuck S, Gwyther S, Mooney M, et al. New response evaluation criteria in solid tumours: revised RECIST guideline (version 1.1). Eur J Cancer. 2009:45:228-47.

19 Winklehner A, Goetti R, Baumueller S, Karlo C, Schmidt B, Raupach R, Flohr $\mathrm{T}$, Frauenfelder T, Alkadhi H. Automated attenuation-based tube potential selection for thoracoabdominal computed tomography angiography: improved dose effectiveness. Invest Radiol. 2011:46:767-73.

20 Eller A, May MS, Scharf M, Schmid A, Kuefner M, Uder M, Lell MM. Attenuation-based automatic kilovolt selection in abdominal computed tomography: effects on radiation exposure and image quality. Invest Radiol. 2012:47:559-65.

21 Gnannt R, Winklehner A, Eberli D, Knuth A, Frauenfelder T, Alkadhi H. Automated tube potential selection for standard chest and abdominal $C T$ in follow-up patients with testicular cancer: comparison with fixed tube potential. Eur Radiol. 2012;22:1937-45.

22 Gordic S, Desbiolles L, Stolzmann P, Gantner L, Leschka S, Husarik DB, Alkadhi $\mathrm{H}$. Advanced modelled iterative reconstruction for abdominal $C T$ : qualitative and quantitative evaluation. Clin Radiol. 2014;69:e497-504.

\section{Submit your next manuscript to BioMed Central and we will help you at every step:}

- We accept pre-submission inquiries

- Our selector tool helps you to find the most relevant journal

- We provide round the clock customer support

- Convenient online submission

- Thorough peer review

- Inclusion in PubMed and all major indexing services

- Maximum visibility for your research

Submit your manuscript at www.biomedcentral.com/submit
Biomed Central 